\title{
Study of charmonia and charmed baryons at Belle
}

\section{Yuji Kato*t}

Kobayashi-Maskawa Institute, Nagoya University, Nagoya 464-8602

E-mail: katodhep1.phys.nagova-u.ac.jp

We report recent results on charmonia and charmed baryons, based on the data collected by the Belle detector located at the KEKB asymmetric-energy $e^{+} e^{-}$collider. These include $B^{+} \rightarrow$ $K^{+} \chi_{c 1} \pi^{+} \pi^{-}$, search for $X Y Z$ in $\Upsilon(1 S)$ decay, precise mass and width measurement of excited $\Xi_{c}$ baryons which decay into $\Lambda D$, and first observation of the doubly Cabibbo suppressed decay of a charmed baryon.

XIII International Conference on Heavy Quarks and Leptons

22-27 May, 2016

Blacksburg, Virginia, USA

${ }^{*}$ Speaker.
†On behalf of the Belle Collaboration 


\section{Introduction}

The observation of $X(3872)$, which does not fit to the conventional charmonium state, by Belle collaboration and successive observation of charmonium-like states so-called $X Y Z$ opened a new era on the hadron spectroscopy. The nature of these states are still not understood and it is very important to study them in many decays and productions.

The charmed baryon is a unique system, which may be interpreted as a heavy charm quark interacting with light di-quark due to the suppression of color spin interaction for the charm quark. The study of charmed baryons leads to the understanding of di-quark degree of freedom.

In this proceedings, recent results on $X Y Z$ states and charmed baryons by Belle collaboration are presented. The data are recorded with the Belle detector located at the KEKB asymmetricenergy $e^{+} e^{-}$collider.

\section{Search for $\chi_{c 1}(2 P)$ and $X(3872)$ in $B^{+} \rightarrow K^{+} \chi_{c 1} \pi^{+} \pi^{-}$decay}

One plausible explanation for the nature of $X(3872)$ is the admixture of molecular $D \bar{D}^{*}$ state and $\chi_{c 1}(2 P)$. This is based on non-observation of the $\chi_{c 1}(2 P)$ and large prompt cross section in the LHC and Tevatron experiments. Therefore, it is important to check the existence of physical $\chi_{c 1}(2 P)$ state. A possible decay mode of the $\chi_{c 1}(2 P)$ is $\chi_{c 1} \pi^{+} \pi^{-}$. Belle collaboration searched for $\chi_{c 1}(2 P)$ and $X(3872)$ in $B^{+} \rightarrow K^{+} \chi_{c 1} \pi^{+} \pi^{-}$together with first observation of exclusive $B$ decays including $\chi_{c 1}$ and $\chi_{c 2}$ decays [四]. The analysis is performed with data sample of $772 \times 10^{6} B \bar{B}$ events.

Figure $\square$ shows the $\Delta E$ distributions for various $B$ meson decays including $\chi_{c 1}$ and $\chi_{c 2}$ with two pions. The statistical significances are $19.2 \sigma(8.4 \sigma), 7.1 \sigma(1.8 \sigma)$ and $6.5 \sigma$ for the $B^{+} \rightarrow$ $\chi_{c 1} \pi^{+} \pi^{-} K^{+}\left(B^{+} \rightarrow \chi_{c 2} \pi^{+} \pi^{-} K^{+}\right), B^{0} \rightarrow \chi_{c 1} \pi^{+} \pi^{-} K_{S}^{0}\left(B^{0} \rightarrow \chi_{c 2} \pi^{+} \pi^{-} K_{S}^{0}\right)$ and $B^{0} \rightarrow \chi_{c 1} \pi^{-} \pi^{0} K^{+}$ $\left(B^{0} \rightarrow \chi_{c 2} \pi^{-} \pi^{0} K^{+}\right)$decay modes, respectively. Figure $\square$ shows the $M\left(\chi_{c 1} \pi^{+} \pi^{-}\right)$distribution for $B^{+} \rightarrow \chi_{c 1} \pi^{+} \pi^{-} K^{+}$candidates. No significant signal of $X(3872)$ and $\chi_{c 1}(2 P)$ are observed. The upper limits for the product of branching fractions: $\mathscr{B}\left(B^{+} \rightarrow X(3872) K^{+}\right) \times \mathscr{B}(X(3872) \rightarrow$ $\left.\chi_{c 1} \pi^{+} \pi^{-}\right)<1.5 \times 10^{-6}$ and $\mathscr{B}\left(B^{+} \rightarrow \chi_{c 1}(2 P) K^{+}\right) \times \mathscr{B}\left(\chi_{c 1}(2 P) \rightarrow \chi_{c 1}(1 P) \pi^{+} \pi^{-}\right)<1.1 \times 10^{-5}$ are obtained ( $90 \%$ C.L.).

\section{Search for $X Y Z$ states in $\Upsilon(1 S)$ inclusive decays}

Most of the $X Y Z$ states have been observed from the decay of $B$ mesons and initial state radiation. To study them in other production modes provides complementary information. Belle collaboration performed the search for $X Y Z$ states from the decay of bottomonium state, $\Upsilon(1 S)$ [[]]. The dominant decay of $\Upsilon(1 S)$ is throught three gluons, which is totally different from $B$ meson decay and initial state radiations. The data size of $\Upsilon(1 S)$ energy region is $5.74 \mathrm{fb}^{-1}$, corresponding to $101 \times 10^{6} \Upsilon(1 S)$ decays. The $X Y Z$ are searched for in fourteen decay modes: $X(3872)$ and $Y(4260)$ to $\pi^{+} \pi^{-} J / \psi ; Y(4260), Y(4360)$ and $Y(4660)$ to $\pi^{+} \pi^{-} \psi(2 S) ; Y(4260)$ to $K^{+} K^{-} J / \psi$; $Y(4140)$ and $X(4350)$ to $\phi J / \psi ; Z_{c}(3900)^{ \pm}, Z_{c}(4200)^{ \pm}$and $Z_{c}(4430)^{ \pm}$to $\pi^{ \pm} J / \psi ; Z_{c}(4050)^{ \pm}$and $Z_{c}(4430)^{ \pm}$to $\pi^{ \pm} \psi(2 S)$; and a predicted $Z_{c s}^{ \pm}$state with mass to $K^{ \pm} J / \psi$. We found no evidence for these decay modes and set upper limits as shown in Table $\mathrm{W}$. 

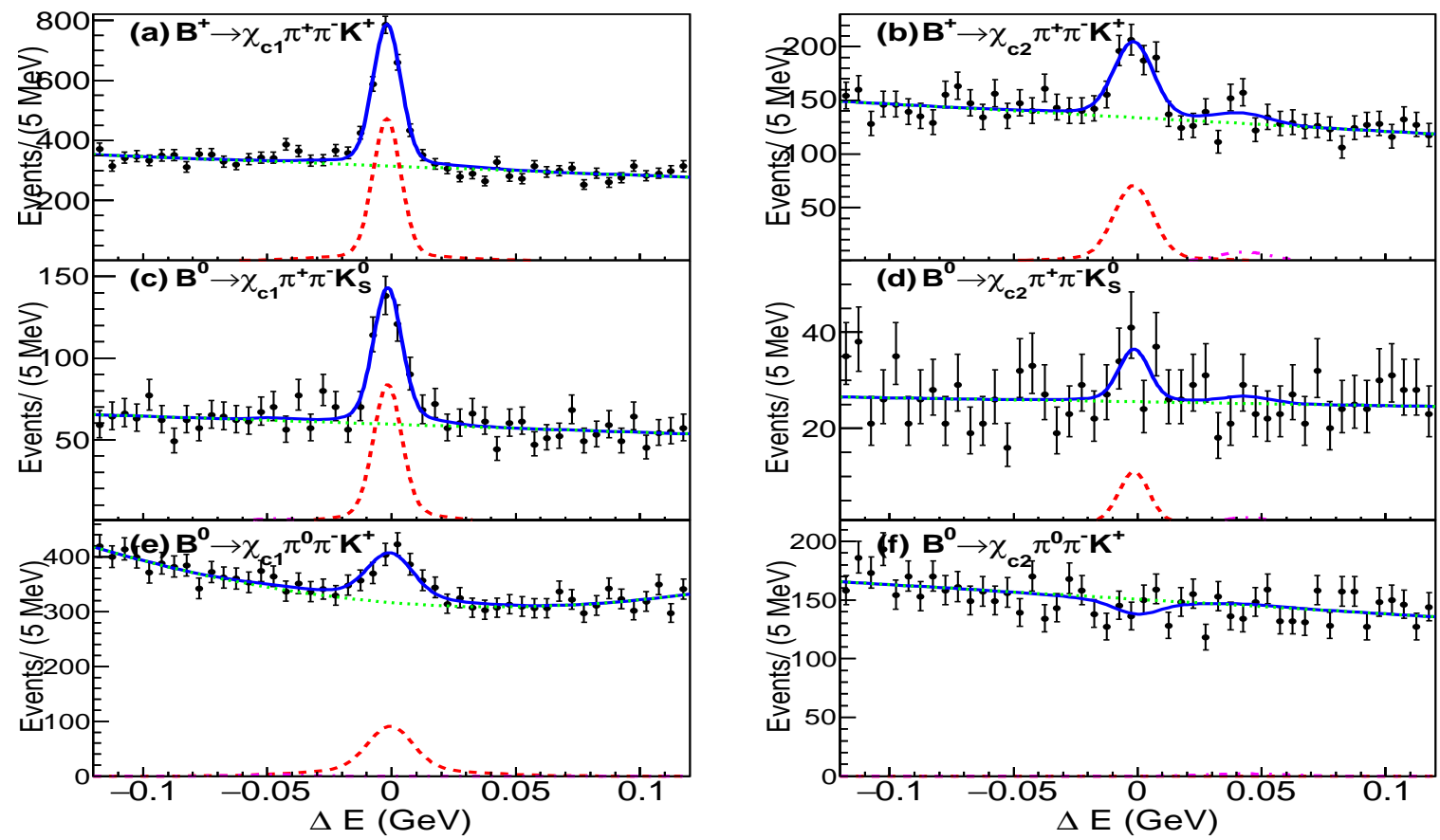

Figure 1: $\Delta E$ distributions for the (a) $B^{+} \rightarrow \chi_{c 1} \pi^{+} \pi^{-} K^{+}$, (b) $B^{+} \rightarrow \chi_{c 2} \pi^{+} \pi^{-} K^{+}$, (c) $B^{0} \rightarrow \chi_{c 1} \pi^{+} \pi^{-} K_{S}^{0}$, (d) $B^{0} \rightarrow \chi_{c 2} \pi^{+} \pi^{-} K_{S}^{0}$, (e) $B^{0} \rightarrow \chi_{c 1} \pi^{0} \pi^{-} K^{+}$and (f) $B^{0} \rightarrow \chi_{c 2} \pi^{0} \pi^{-} K^{+}$decay modes. The curves show the signal (red dashed), peaking background (magenta dash-dotted) and the background component (green dotted for combinatorial) as well as the overall fit (blue solid).

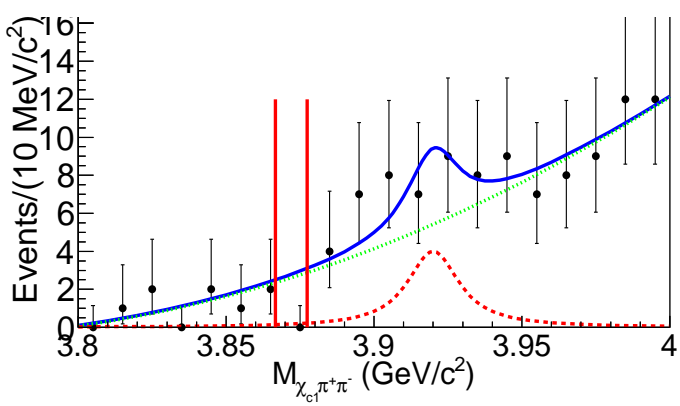

Figure 2: The $\chi_{c 1} \pi^{+} \pi^{-}$invariant mass spectrum for $B^{+} \rightarrow \chi_{c 1} \pi^{+} \pi^{-} K^{+}$candidates. Two vertical red lines show the $\pm 3 \sigma$ window to search for $X(3872) \rightarrow \chi_{c 1} \pi^{+} \pi^{-}$. The curves show the $\chi_{c 1}(2 P)$ signal (red dashed) and the background (green dotted) and the overall fit (blue solid). 
Table 1: Summary of the upper limits on the $\Upsilon(1 S)$ inclusive decays into the exotic charmoniumlike states $X Y Z$, where $N_{\text {fit }}$ is the number of fitted signal events, $N_{\text {up }}$ is the upper limit on the number of signal events taking into account systematic errors, $\varepsilon$ is the reconstruction efficiency, $\sigma_{\text {syst }}$ is the total systematic uncertainty, $\Sigma$ is the signal significance with systematic errors included, and $\mathscr{B}_{R}^{\text {prod }}=\mathscr{B}(\Upsilon(1 S) \rightarrow X Y Z+$ anything $\mathscr{B}(X Y Z \rightarrow J / \psi(\psi(2 S))+$ hadrons $)$ is the measured product branching fraction at the $90 \%$ C.L.

\begin{tabular}{lrrrccc}
\hline \hline State & \multicolumn{1}{c}{$N_{\text {fit }}$} & \multicolumn{1}{c}{$N_{\text {up }}$} & $\varepsilon(\%)$ & $\sigma_{\text {syst }}(\%)$ & $\Sigma(\sigma)$ & $\mathscr{B}_{R}^{\text {prod }}$ \\
\hline$X(3872) \rightarrow \pi^{+} \pi^{-} J / \psi$ & $4.8 \pm 15.4$ & 31.4 & 3.26 & 18.7 & 0.3 & $<9.5 \times 10^{-6}$ \\
$Y(4260) \rightarrow \pi^{+} \pi^{-} J / \psi$ & $-31.1 \pm 88.9$ & 134.6 & 3.50 & 35.6 & - & $<3.8 \times 10^{-5}$ \\
$Y(4260) \rightarrow \pi^{+} \pi^{-} \psi(2 S)$ & $6.7 \pm 29.4$ & 56.9 & 0.71 & 35.0 & 0.2 & $<7.9 \times 10^{-5}$ \\
$Y(4360) \rightarrow \pi^{+} \pi^{-} \psi(2 S)$ & $-25.4 \pm 30.1$ & 45.6 & 0.86 & 50.0 & - & $<5.2 \times 10^{-5}$ \\
$Y(4660) \rightarrow \pi^{+} \pi^{-} \psi(2 S)$ & $-55.0 \pm 26.2$ & 23.1 & 1.06 & 40.7 & - & $<2.2 \times 10^{-5}$ \\
$Y(4260) \rightarrow K^{+} K^{-} J / \psi$ & $-13.7 \pm 10.9$ & 14.5 & 1.91 & 45.8 & - & $<7.5 \times 10^{-6}$ \\
$Y(4140) \rightarrow \phi J / \psi$ & $-0.1 \pm 1.2$ & 3.6 & 0.69 & 11.0 & - & $<5.2 \times 10^{-6}$ \\
$X(4350) \rightarrow \phi J / \psi$ & $2.3 \pm 2.5$ & 7.6 & 0.92 & 10.4 & 1.2 & $<8.1 \times 10^{-6}$ \\
$Z_{c}(3900)^{ \pm} \rightarrow \pi^{ \pm} J / \psi$ & $-26.5 \pm 39.1$ & 57.5 & 4.39 & 47.3 & - & $<1.3 \times 10^{-5}$ \\
$Z_{c}(4200)^{ \pm} \rightarrow \pi^{ \pm} J / \psi$ & $-238.6 \pm 154.2$ & 235.1 & 3.87 & 48.4 & - & $<6.0 \times 10^{-5}$ \\
$Z_{c}(4430)^{ \pm} \rightarrow \pi^{ \pm} J / \psi$ & $94.2 \pm 71.4$ & 195.8 & 3.97 & 34.4 & 1.2 & $<4.9 \times 10^{-5}$ \\
$Z_{c}(4050)^{ \pm} \rightarrow \pi^{ \pm} \psi(2 S)$ & $37.0 \pm 47.7$ & 112.7 & 1.27 & 46.2 & 0.4 & $<8.8 \times 10^{-5}$ \\
$Z_{c}(4430)^{ \pm} \rightarrow \pi^{ \pm} \psi(2 S)$ & $23.2 \pm 42.4$ & 92.0 & 1.35 & 47.1 & 0.1 & $<6.7 \times 10^{-5}$ \\
$Z_{c s}^{ \pm} \rightarrow K^{ \pm} J / \psi$ & $-22.2 \pm 17.4$ & 22.4 & 3.88 & 48.7 & - & $<5.7 \times 10^{-6}$ \\
\hline \hline
\end{tabular}

\section{Precise measurements of masses and widths of excited $\Xi_{c}$ baryons}

The mass difference of charged and neutral $\Xi_{c}$ baryons (isospin splitting) is comes from the mass difference of up and down quarks and difference of electron-magnetic interactions. Therefore measurement of the isospin splitting provides a good information to deduce the wave function of $\Xi_{c}$ baryons. Belle collaboration performed the measurement of masses and widths of five excited $\Xi_{c}$ baryons decaying into $\Xi_{c}^{+}$or $\Xi_{c}^{0}: \Xi_{c}^{\prime}, \Xi_{c}(2645), \Xi_{c}(2790), \Xi_{c}(2815)$, and $\Xi_{c}(2980)$ [3]]. The analysis is performed with data sample of $980 \mathrm{fb}^{-1}$ taken with various energies. The $\Xi_{c}^{+}$and $\Xi_{c}^{0}$ are reconstructed from 7 and 10 decay modes and yields are about $1 \times 10^{5}$ and $5 \times 10^{4}$. Table $\square$ summarizes the measurement of masses and widths for $5 \Xi_{c}$ isospin doublets. The precisions for masses are improved from world average by about one order of magnitude. For the widths, first significant measurements have been done for many states. Table [] summarizes the measurement of isospin splittings. We find absolute values of isospin splittings are small for $\Xi_{c}^{\prime}$ and $\Xi_{c}(2645)$ and large for other three states. These may reflect the difference of internal structure of these charmed baryons.

\section{Excited $\Xi_{c}$ baryons decaying into $\Lambda D$ final states}

In the higher excited regions, there are couple of past studies. Belle and BaBar collaboration reported observation of $\Xi_{c}(2980)^{+}, \Xi_{c}(3055)^{+}$, and $\Xi_{c}(3080)^{+}$decaying into $\Sigma_{c}^{++} K^{-}$final state [[G, 目] and $\Xi_{c}(3080)^{+}$in $\Sigma_{c}^{*++}$ final state. Belle collaboration newly performed a study of excited $\Xi_{c}$ baryons decaying into $\Lambda D$ final states. 
Table 2: Result for masses (in $\mathrm{MeV} / \mathrm{c}^{2}$ ) and widths (in $\mathrm{MeV}$ ) measurements for the five isodoublets under study. For comparison, the 2015 world averages (denoted "PDG") are also quoted. Mass differences are with respect to the daughter states.

\begin{tabular}{|c|c|c|c|c|}
\hline Particle & Mass & $M-M\left(\Xi_{c}\right)$ & $M-M\left(\Xi_{c}^{\prime}\right)$ & Width \\
\hline$\Xi_{c}(2645)^{+}$ & $2645.58 \pm 0.06 \pm 0.07_{-0.40}^{+0.28}$ & $174.66 \pm 0.06 \pm 0.07$ & & $2.06 \pm 0.13 \pm 0.13$ \\
\hline PDG & $2645.9 \pm 0.5$ & $175.0 \pm 0.6$ & & $2.6 \pm 0.2 \pm 0.4$ \\
\hline$\Xi_{c}(2645)^{0}$ & $2646.43 \pm 0.07 \pm 0.07_{-0.40}^{+0.28}$ & $178.46 \pm 0.07 \pm 0.07$ & & $2.35 \pm 0.18 \pm 0.13$ \\
\hline PDG & $2645.9 \pm 0.5$ & $178.0 \pm 0.6$ & & $<5.5$ \\
\hline$\Xi_{c}(2815)^{+}$ & $2816.73 \pm 0.08 \pm 0.06_{-0.40}^{+0.28}$ & $348.80 \pm 0.08 \pm 0.06$ & & $2.43 \pm 0.20 \pm 0.17$ \\
\hline PDG & $2816.6 \pm 0.9$ & $348.7 \pm 0.9$ & & $<3.5$ \\
\hline$\Xi_{c}(2815)^{0}$ & $2820.20 \pm 0.08 \pm 0.07_{-0.40}^{+0.28}$ & $349.35 \pm 0.08 \pm 0.07$ & & $2.54 \pm 0.18 \pm 0.17$ \\
\hline PDG & $2819.6 \pm 1.2$ & $348.8 \pm 1.2$ & & $<6.5$ \\
\hline$\Xi_{c}(2980)^{+}$ & $2966.0 \pm 0.8 \pm 0.2_{-0.4}^{+0.3}$ & $498.1 \pm 0.8 \pm 0.2$ & & $28.1 \pm 2.4_{-5.0}^{+1.0}$ \\
\hline PDG & $2970.7 \pm 2.2$ & & & $17.9 \pm 3.5$ \\
\hline$\Xi_{c}(2980)^{0}$ & $2970.8 \pm 0.7 \pm 0.2_{-0.4}^{+0.3}$ & $499.9 \pm 0.7 \pm 0.2$ & & $30.3 \pm 2.3_{-1.8}^{+1.0}$ \\
\hline PDG & $2968.0 \pm 2.6 \pm 0.5$ & & & $20 \pm 7$ \\
\hline$\Xi_{c}^{\prime+}$ & $2578.4 \pm 0.1 \pm 0.4_{-0.4}^{+0.3}$ & $110.5 \pm 0.1 \pm 0.4$ & & \\
\hline PDG & $2575.6 \pm 3.0$ & $107.8 \pm 3.0$ & & \\
\hline$\Xi_{c}^{\prime 0}$ & $2579.2 \pm 0.1 \pm 0.4_{-0.4}^{+0.3}$ & $108.3 \pm 0.1 \pm 0.4$ & & \\
\hline PDG & $2577.9 \pm 2.9$ & $107.0 \pm 2.9$ & & \\
\hline$\Xi_{c}(2790)^{+}$ & $2791.6 \pm 0.2 \pm 0.1 \pm 0.4_{-0.4}^{+0.3}$ & $320.7 \pm 0.2 \pm 0.1 \pm 0.4$ & $213.2 \pm 0.2 \pm 0.1$ & $8.9 \pm 0.6 \pm 0.8$ \\
\hline PDG & $2789.8 \pm 3.2$ & $318.2 \pm 3.2$ & & $<15$ \\
\hline$\Xi_{c}(2790)^{0}$ & $2794.9 \pm 0.3 \pm 0.1 \pm 0.4_{-0.4}^{+0.3}$ & $323.8 \pm 0.2 \pm 0.1 \pm 0.4$ & $215.7 \pm 0.2 \pm 0.1$ & $10.0 \pm 0.7 \pm 0.8$ \\
\hline PDG & $2791.9 \pm 3.3$ & $324.0 \pm 3.3$ & & $<12$ \\
\hline
\end{tabular}

Table 3: The isospin splitting between the members of each isodoublet.

\begin{tabular}{|l|c|}
\hline \hline Particle & $M\left(\Xi_{c}^{+}\right)-M\left(\Xi_{c}^{0}\right)\left(\mathrm{MeV} / c^{2}\right)$ \\
\hline$\Xi_{c}(2645)$ & $-0.85 \pm 0.09 \pm 0.08 \pm 0.48$ \\
$\Xi_{c}(2815)$ & $-3.47 \pm 0.12 \pm 0.05 \pm 0.48$ \\
$\Xi_{c}(2980)$ & $-4.8 \pm 0.1 \pm 0.2 \pm 0.5$ \\
$\Xi_{c}^{\prime}$ & $-0.8 \pm 0.1 \pm 0.1 \pm 0.5$ \\
$\Xi_{c}(2790)$ & $-3.3 \pm 0.4 \pm 0.1 \pm 0.5$ \\
\hline \hline
\end{tabular}

Figure B] shows the $M(\Lambda D)$ distributions. In the $\Lambda D^{+}$final state, we can see a peak corresponding to $\Xi_{c}(3055)^{+}$and $\Xi_{c}(3080)^{+}$. In the $\Lambda D^{0}$ final state, we can see peaks corresponding to $\Xi_{c}(3055)^{0}$ in all the $D^{0}$ modes. The statistical significance of the peak is $8.6 \sigma$. This is the first observation of $\Xi_{c}(3055)^{0}$. The mass and width of the $\Xi_{c}(3055)^{0}$ are measured to be (3059.0 $\pm 0.5 \pm 0.6) \mathrm{MeV} / c^{2}$ and $(6.4 \pm 2.1 \pm 1.1) \mathrm{MeV}$, respectively. A combined analysis of these particles by comparing their decays into $\Lambda D^{+}$with those into $\Sigma_{c}^{++} K^{-}$and $\Sigma_{c}^{*++} K^{-}$is also performed. The ratios of branching fractions, $\mathscr{B}\left(\Xi_{c}(3055)^{+} \rightarrow \Lambda D^{+}\right) / \mathscr{B}\left(\Xi_{c}(3055)^{+} \rightarrow \Sigma_{c}^{++} K^{-}\right)=$ $5.09 \pm 1.01 \pm 0.76, \mathscr{B}\left(\Xi_{c}(3080)^{+} \rightarrow \Lambda D^{+}\right) / \mathscr{B}\left(\Xi_{c}(3080)^{+} \rightarrow \Sigma_{c}^{++} K^{-}\right)=1.29 \pm 0.30 \pm 0.15$, and 
$\mathscr{B}\left(\Xi_{c}(3080)^{+} \rightarrow \Sigma_{c}^{*++} K^{-}\right) / \mathscr{B}\left(\Xi_{c}(3080)^{+} \rightarrow \Sigma_{c}^{++} K^{-}\right)=1.07 \pm 0.27 \pm 0.04$, are obtained. These information are useful to understand the nature of these baryons.
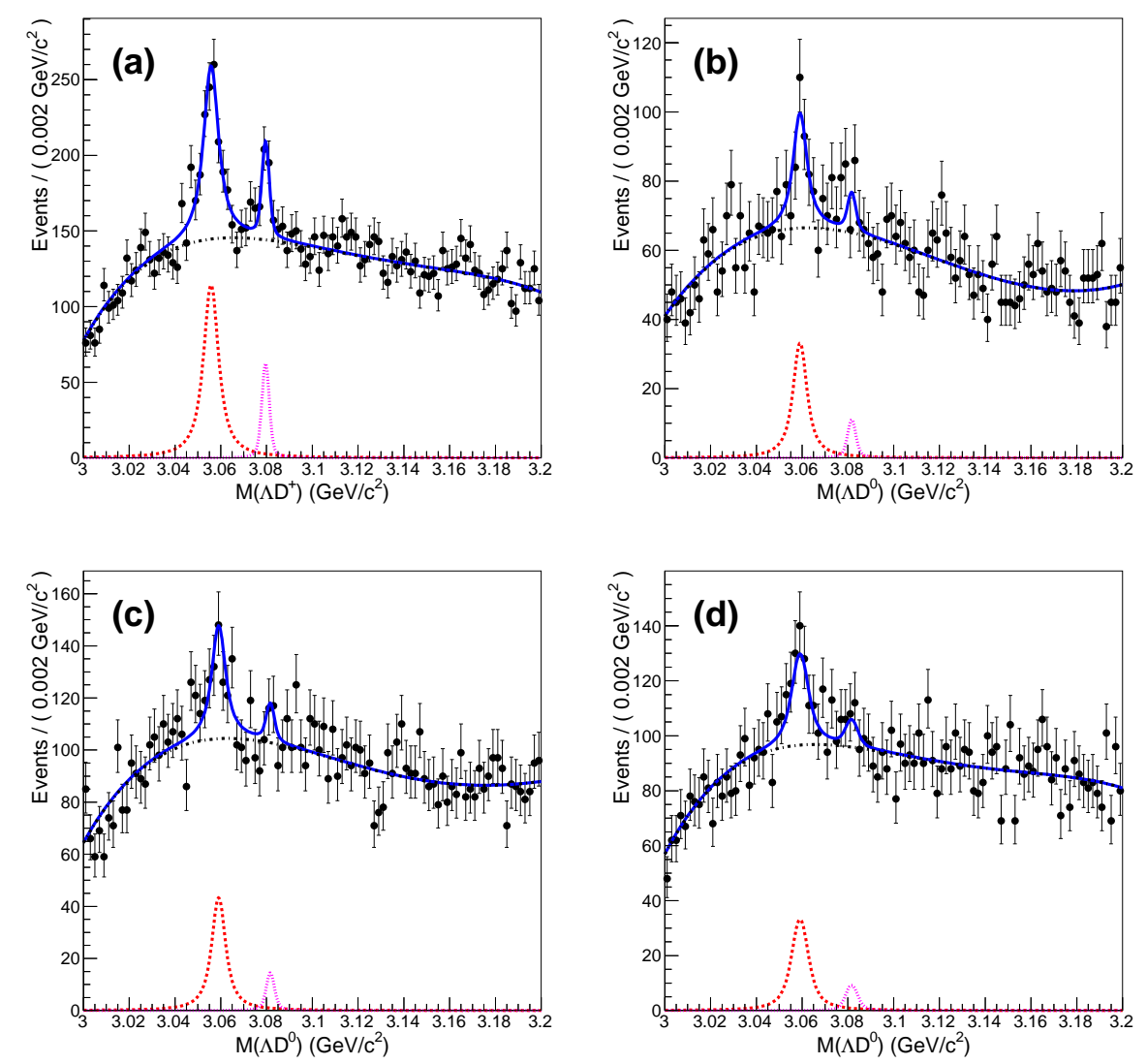

Figure 3: $M(\Lambda D)$ distributions. Points with statistical error bars are data. Blue solid lines show the fit results. The red dashed, magenta dotted, and black dashed-dotted lines show the $\Xi_{c}(3055)$ signal, the $\Xi_{c}(3080)$ signal, and the background components, respectively. (a) $M\left(\Lambda D^{+}\right)$distribution; $M\left(\Lambda D^{0}\right)$ distributions for the (b) $K^{-} \pi^{+}$, (c) $K^{-} \pi^{+} \pi^{+} \pi^{-}$, and (d) $K^{-} \pi^{+} \pi^{0} D^{0}$ decay modes.

\section{First observation of Doubly Cabibbo-Suppressed decay of a charmed baryon: $\Lambda_{c}^{+} \rightarrow p K^{+} \pi^{-}$}

In the baryon sector, there had been no observation of the Doubly Cabbibo-Suppressed (DCS) decay. Belle collaboration reported the first observation of such decay $\Lambda_{c}^{+} \rightarrow p K^{+} \pi^{-}$[四]. Figure Q 1 shows the distribution of $\mathrm{M}\left(p K^{+} \pi^{+}\right)$. We can see clear peak corresponding to DCS decay. The significance of the peak after subtracting the contribution from $\Lambda_{c}^{+} \rightarrow \Lambda K^{+} \rightarrow p K^{+} \pi^{-}$is

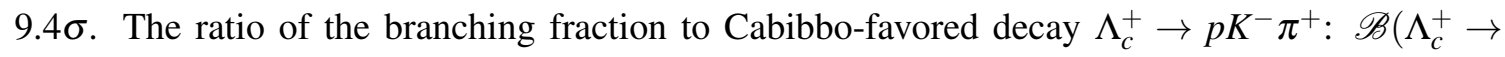
$\left.p K^{+} \pi^{-}\right) / \mathscr{B}\left(\Lambda_{c}^{+} \rightarrow p K^{-} \pi^{+}\right)$, is $(2.35 \pm 0.27 \pm 0.21) \times 10^{-3}$, which is consistent with $\tan \left(\theta_{c}\right)$, where $\theta_{c}$ is the Cabibbo-angle. 


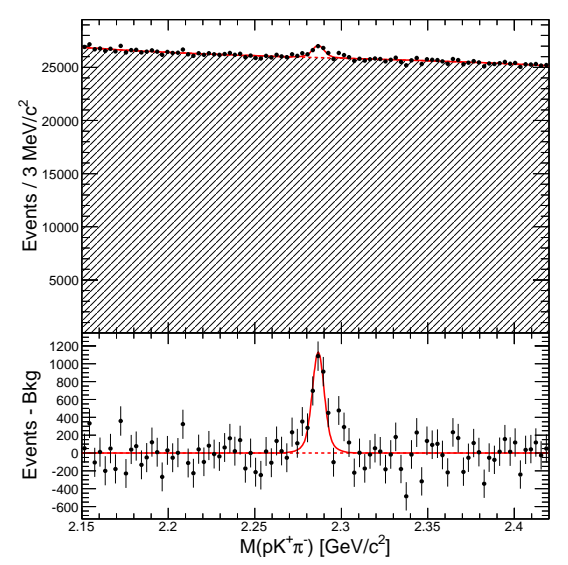

Figure 4: Distribution of $M\left(p K^{+} \pi^{-}\right)$(top) and residuals of data with respect to the fitted combinatorial background (bottom). The curves indicate the fit result: the full fit model (solid) and the combinatoric background only (dashed).

\section{Conclusion}

Belle collaboration has been actively publishing the results on charmonium and charmed baryons after the end of data taking in 2010. Study of various production and decay for $X Y Z$ are presented. Precise mass and width measurements and study of $\Lambda D$ final state for excited $\Xi_{c}$ baryons are presented. Finally, first observation of DCS decay of a baryon: $\Lambda_{c}^{+} \rightarrow p K^{+} \pi^{-}$is presented.

\section{Acknowledgments}

This work is supported by a Grant-in-Aid for Scientific Research (S) "Probing New Physics with Tau-Lepton" (No.26220706) and Grant-in-Aid for Scientific Research on Innovative Areas "Elucidation of New Hadrons with a Variety of Flavors".

\section{References}

[1] V. Bhardwaj et al. [Belle Collaboration], Phys. Rev. D 93, no. 5, 052016 (2016) doi:10.1103/PhysRevD.93.052016 [arXiv:1512.02672 [hep-ex]].

[2] C. P. Shen et al. [Belle Collaboration], Phys. Rev. D 93, no. 11, 112013 (2016) doi:10.1103/PhysRevD.93.112013 [arXiv:1605.00990 [hep-ex]].

[3] J. Yelton et al. [Belle Collaboration], arXiv:1607.07123 [hep-ex].

[4] R. Chistov et al. [Belle Collaboration], Phys. Rev. Lett. 97, 162001 (2006) [hep-ex/0606051].

[5] B. Aubert et al. [BaBar Collaboration], Phys. Rev. D 77, 012002 (2008) [arXiv:0710.5763 [hep-ex]].

[6] Y. Kato et al. [Belle Collaboration], Phys. Rev. D 89, 052003 (2014) [arXiv:1312.1026 [hep-ex]].

[7] Y. Kato et al. [Belle Collaboration], Phys. Rev. D 94, no. 3, 032002 (2016) doi:10.1103/PhysRevD.94.032002 [arXiv:1605.09103 [hep-ex]]. 
[8] S. B. Yang et al. [Belle Collaboration], Phys. Rev. Lett. 117, no. 1, 011801 (2016) doi:10.1103/PhysRevLett.117.011801 [arXiv:1512.07366 [hep-ex]]. 